\title{
Molecular autopsy by trio exome sequencing (ES) and postmortem examination in fetuses and neonates with prenatally identified structural anomalies
}

\author{
Elizabeth Quinlan-Jones, MSc $c^{1,2}$, Jenny Lord, $\mathrm{PhD}^{3}$, Denise Williams, FRCP MSc ${ }^{1}$, Sue Hamilton, BSc ${ }^{4}$, \\ Tamas Marton, FRCPath PhD ${ }^{5}$, Ruth Y. Eberhardt, $\mathrm{PhD}^{3}$, Gabriele Rinck, PhD ${ }^{3}$, Elena Prigmore, $\mathrm{PhD}^{3}$, \\ Rebecca Keelagher, $\mathrm{PhD}^{4}$, Dominic J. McMullan, FRCPath ${ }^{4}$, Eamonn R. Maher, MD FRCP ${ }^{6}$, \\ Matthew E. Hurles, PhD FMedSci ${ }^{3}$ and Mark D. Kilby, DSc MD ${ }^{2,7}$
}

Purpose: To determine the diagnostic yield of combined exome sequencing (ES) and autopsy in fetuses/neonates with prenatally identified structural anomalies resulting in termination of pregnancy, intrauterine, neonatal, or early infant death.

Methods: ES was undertaken in 27 proband/parent trios following full autopsy. Candidate pathogenic variants were classified by a multidisciplinary clinical review panel using American College of Medical Genetics and Genomics (ACMG) guidelines.

Results: A genetic diagnosis was established in ten cases (37\%). Pathogenic/likely pathogenic variants were identified in nine different genes including four de novo autosomal dominant, three homozygous autosomal recessive, two compound heterozygous autosomal recessive, and one X-linked. KMT2D variants (associated with Kabuki syndrome postnatally) occurred in two cases. Pathogenic variants were identified in $5 / 13$ (38\%) cases with multisystem anomalies, in $2 / 4(50 \%)$ cases with fetal akinesia deformation sequence, and in $1 / 4$ (25\%) cases each with cardiac and brain anomalies and hydrops fetalis. No pathogenic variants were detected in fetuses with genitourinary (1), skeletal (1), or abdominal (1) abnormalities.

Conclusion: This cohort demonstrates the clinical utility of molecular autopsy with ES to identify an underlying genetic cause in structurally abnormal fetuses/neonates. These molecular findings provided parents with an explanation of the developmental abnormality, delineated the recurrence risks, and assisted the management of subsequent pregnancies.

Genetics in Medicine (2019) 21:1065-1073; https://doi.org/10.1038/s41436018-0298-8

Keywords: exome sequencing; fetuses; neonates; autopsy; genetic diagnosis

\section{INTRODUCTION}

Fetal structural anomalies (FSAs) complicate $3 \%$ of pregnancies and range in phenotype from isolated minor anomalies to severe multisystem abnormalities, many of which are associated with high perinatal mortality rates, or contribute to long-term morbidity. ${ }^{1}$ Currently, such pregnancies may be investigated prenatally by chorionic villus sampling (CVS) or amniocentesis, to obtain fetal DNA for quantitative fluorescent-polymerase chain reaction (QF-PCR) to exclude common autosomal and sex chromosome aneuploidies, and chromosomal microarray (CMA) to detect submicroscopic copy-number variations (CNVs). ${ }^{2,3}$ Adding CMA analysis to G-banded karyotyping increases the detection rate in this group of fetuses by up to $5 \%\left(\right.$ ref. $\left.^{4}\right)$. However, no identifiable genetic cause is detected in over $60 \%$ of cases. ${ }^{5}$ If the pregnancy is terminated or fetal demise occurs, autopsy is important because despite generally good correlation between prenatal ultrasound and autopsy findings, the latter can identify additional subtle malformations and may elucidate further information allowing an etiologic diagnosis necessary for accurate genetic counseling. ${ }^{6,7}$

Exome sequencing (ES) is an established diagnostic tool in delineating the genetic etiology of congenital abnormalities and neurodevelopmental disorders ${ }^{8,9}$ in adults and children. The use of ES for the analysis of prenatally obtained fetal DNA has been reported in small cohort series and a recent

${ }^{1}$ Department of Clinical Genetics, Birmingham Women's \& Children's NHS Foundation Trust, Birmingham, UK; ${ }^{2}$ West Midlands Fetal Medicine Centre, Birmingham Women's \& Children's NHS Foundation Trust, Birmingham, UK; ${ }^{3}$ Wellcome Sanger Institute, Hinxton, Cambridge, UK; ${ }^{4}$ West Midlands Regional Genetics Service, Birmingham Women's and Children's Hospital NHS Foundation Trust, Birmingham, UK; ${ }^{5}$ West Midlands Regional Perinatal Pathology Service, Birmingham Women's and Children's NHS Foundation Trust, Birmingham, UK; ${ }^{6}$ Department of Medical Genetics, University of Cambridge and NIHR Cambridge Biomedical Research Centre, Cambridge, UK; ${ }^{7}$ Institute of Metabolism and Systems Research, College of Medical and Dental Sciences, University of Birmingham, Birmingham, UK. Correspondence: Mark D. Kilby (M.D.Kilby@bham.ac.uk)

These authors contributed equally: Elizabeth Quinlan-Jones and Jenny Lord

These are equal senior authors: Eamonn R. Maher, Matthew E. Hurles, and Mark D. Kilby 
review has indicated that this technology reveals pathogenic findings in a range of between $6.2 \%$ and $80 \%$ of cases. ${ }^{10}$ Our group has reported prospective data on 610 prenatal cases with a spectrum of fetal anomalies detected by ultrasound scan (USS) and demonstrated a diagnostic yield of 8.5\% (95\% confidence interval [CI]: $6.4-11.0 \%$ ); a further $3.9 \%$ had a variant of uncertain significance (VUS) with potential clinical value. ${ }^{11}$

One of the limitations in the use of prenatal ES is a lack of accurate fetal phenotyping using prenatal ultrasonography alone. ${ }^{10}$ In the cases of FSA ending in fetal demise, postnatal dysmorphological examination and autopsy in addition to prenatal ultrasound might be predicted to improve the interpretation of genetic findings and increase diagnostic yield. We have evaluated this in a cohort of fetuses/neonates $(n=27)$. We correlated the results of ES from proband-parent trios with prenatal USS and autopsy findings to determine the clinical utility of molecular autopsy. Our study supports the use of trio ES and autopsy to identify the underlying etiology of structural anomalies, expand knowledge of the genetic basis of fetal development, and enable improved descriptions of phenotypic variation of known genetic disorders. ${ }^{12}$

\section{Recruitment of cases}

\section{MATERIALS AND METHODS}

Parents of fetuses/neonates $(n=27)$ with a significant structural anomaly resulting in termination of pregnancy (TOP), intrauterine fetal demise (IUFD), or neonatal/infant death (NND/ID) were identified by clinical geneticists, perinatal pathologists, and fetal medicine specialists at Birmingham Women's and Children's NHS Foundation Trust (BWCNFT) and recruited to this study between May 2015 and December 2017. Prospectively obtained fetal DNA at autopsy was stored at West Midlands Regional Genetics Laboratory (WMRGL) and, with written consent, trio (proband/biparental) ES was performed at the Wellcome Sanger Institute (WSI) in Cambridge, United Kingdom. All cases had previously undergone standard testing for aneuploidy (QF-PCR) and whole-genome copy-number analysis by CMA with no pathogenic abnormalities found. Parents were prospectively informed that only results relevant to the USS-detected FSA would be reported back (i.e., not secondary findings). Ethics approval to undertake the research was granted by National Research Ethics Service (NRES) Committee West Midlands-South Birmingham (ref: 13/WM/ 1219). NHS Trust approval was provided by BWCNFT-Research and Development Department.

\section{Exome sequencing, variant calling, and annotation}

In 26 cases DNA was extracted directly from fetal tissue obtained at autopsy, and in one case fetal DNA was extracted from cultured amniocytes obtained prenatally. The autopsy tissue used to extract DNA varied from case to case and included skin, lung, liver, and muscle. Parental DNA was obtained from blood or saliva samples to form proband/ parent trios. Trio DNA samples were batched at WMRGL and transferred to the WSI (Cambridge, UK) for ES through the PAGE Study ${ }^{13}$ sequencing pipeline. For full information on sequencing and analysis see Supplementary Methods. Briefly, $125 \mathrm{ng}$ genomic DNA was fragmented ( 150 bp) and library preparation was conducted following Illumina's standard methodologies. Indexed libraries were pooled and exome capture undertaken with the Agilent SureSelect XT Human All Exon V5 Plus with custom ELID\#0337431 (Agilent Technologies, Santa Clara, CA, USA). Seventy-five base paired-end sequencing (with six samples per lane on Illumina HiSeq 2500) was conducted following the manufacturer's instructions, with over $97 \%$ of exonic positions having depth of coverage $>13 \times$. Mapping was conducted with the Burrows-Wheeler Aligner (BWA, version 0.59). Singlenucleotide variants (SNVs) and indels were identified using GATK HaplotypeCaller version 3.6 (ref. ${ }^{14}$ ), while DeNovoGear ${ }^{15}$ was used to identify de novo variants, and CoNVex (http://www.uk10k.org/assets/ashg_vijayarangakannan_etal_ 2012.pdf) and CIFER (https://github.com/jeremymcrae/cifer) were used to identify and assess the inheritance of CNVs. Variant call format (VCF) files were annotated using Ensembl's Variant Effect Predictor (VEP). ${ }^{16}$ Variants were filtered to identify those of potential clinical significance based on functional consequence (i.e., protein altering variants) and minor allele frequency (MAF) across a number of resources (see Supplementary Methods) (MAF $>0.005$ excluded, with more stringent filters applied for variants in monoallelic genes [MAF $>0.0005$ excluded if both parents' data present]). The analysis pipeline produced a selection of candidate pathogenic variants with an inheritance pattern consistent with diseases associated with the gene in question, and were filtered using a modified developmental disorder-associated gene panel. A virtual gene panel was used to maximize diagnostic yield and minimize the potential for secondary findings, and high numbers of variants of uncertain significance. This modified gene panel included the full DDG2P gene list ${ }^{17}$ (https://www. ebi.ac.uk/gene2phenotype) with several genes removed due to the absence of a prenatal phenotype, as well as some additional genes known to be associated with fetal development sourced from the literature (see Supplementary Table 1). Sequencing data is available from the European Genomephenome Archive (https://www.ebi.ac.uk/ega/).

\section{Variant interpretation and classification}

A multidisciplinary clinical review panel (CRP) comprising clinical geneticists, fetal medicine specialists, perinatal pathologists, clinical scientists, midwives, and bioinformaticians reviewed and classified candidate pathogenic variants. ${ }^{11}$ Through consensus agreement variants were classified using the American College of Medical Genetics and Genomics (ACMG) guidelines. ${ }^{18}$ Pathogenic and likely pathogenic variants were further classified as to whether they explained the observed phenotype fully, partially, or did not explain the phenotype. ${ }^{18}$ The autopsies were carried out at West Midlands Regional Perinatal Pathology Service (located 
within a tertiary, teaching hospital setting) by designated subspecialty perinatal pathologists after informed consent was sought from parents. In all cases a full autopsy was performed according to Royal College of Pathologists guidelines, ${ }^{19,20}$ which included external examination, X-ray, comprehensive internal examination, and histology of relevant internal organs. Abnormal findings were photographically documented and discussed with senior clinical geneticists at a regular dysmorphology meeting. Pathogenic/likely pathogenic variants assessed to be causative for the observed fetal phenotype were confirmed by Sanger sequencing. Reports were issued to the clinical geneticist/fetal medicine specialist involved in the care of the family to return the findings.

\section{Statistical analysis}

Fisher's exact test was used to compare the diagnostic rates and the number of homozygous autosomal recessive diagnoses between cases with and without self-reported consanguinity using $\mathrm{R}$ (version 3.1.3).

\section{Demographic characteristics}

\section{RESULTS}

The median age of women recruited was 30 years $(95 \% \mathrm{CI}$ 27.4-31.4). Nine (33\%) women were nulliparous and 18 (67\%) were multiparous. Gestational age (GA) at delivery ranged from 15 to 35 weeks (median GA 23 weeks) (95\% CI 21.4-28.8). In five pregnancies (19\%) the women had a previous history of a baby with a congenital malformation and in six pregnancies $(22 \%)$ there was parental consanguinity. There were 15 (55\%) TOP, 8 (30\%) IUFD, and 4 (15\%) NND/ ID. Gestational age ranged from 15 to 35 weeks (TOP), 16 to 34 weeks (IUFD), and 0 to 58 days postnatal (NND/ID). There was no correlation between the presence of a diagnostic ES finding and maternal age, ethnicity, parity, or GA. Pregnancy outcome per proband according to phenotype class (determined at postmortem [PM] examination) is presented in Supplementary Figure 1.

\section{Phenotype classification}

Phenotypic information was obtained from the prenatal imaging (USS and fetal magnetic resonance image [MRI]) reports, and from the final autopsy report. Initially, the 27 probands ( 10 female, 17 male) were categorized by phenotype into 12 classes according to the anatomical system affected, jointly by the fetal medicine specialist and clinical geneticist at the study site. Following this, the perinatal pathologist also independently categorized the cases according to the same criteria (Tables 1 and 2). This resulted in the reclassification of 4 fetal phenotypes (15\%), increasing the number of brain (1 to 3 ) and abdominal (0 to 1 ) phenotypes, and reducing the number of fetal akinesia deformation sequence (FADS) (4 to 3 ), genitourinary (2 to 1), and multisystem (14 to 13) phenotypes; the cases of cardiac (4), skeletal (1), and hydrops fetalis (1) remained unchanged. Reclassification of four cases by the perinatal pathologist according to the autopsy findings occurred for the following indications. Case 12 (see Table 2) was an isolated omphalocele with no associated ventriculomegaly (VM) noted at autopsy and was therefore reclassified as an isolated abdominal malformation. Prenatally it was reported that the lateral ventricles measured just over $10 \mathrm{~mm}$ bilaterally inferring "borderline" VM as an ultrasound finding. It is possible that the VM was present and subsequently resolved with advancing gestation. However, it has been described that autopsy may fail to confirm VM identified prenatally in approximately $50 \%$ of cases due to resolution rather than autopsy artifact. ${ }^{21}$ Case 15 had a brain abnormality identified prenatally (see Table 2), and subtle skull, facial, and cardiac abnormalities confirmed at autopsy. It was concluded that the additional anomalies observed postnatally were subsequent to the primary brain anomaly and thus the phenotype was reclassified from multisystem to brain. The autopsy examination for case 19 (see Table 2) identified internal genital and vertebral anomalies in addition to the renal malformations diagnosed on prenatal ultrasound, and hence it was reclassified from renal to multisystem. Finally in case 23 (see Table 2) the observed FADS was determined as having been caused by an underlying central nervous system abnormality and was therefore reclassified to the brain abnormality group. Almost half (13 cases) were classified as having structural anomalies affecting multiple organ systems. The remaining were classified as cardiac (4), FADS (3), brain (3), genitourinary (1), skeletal (1), abdominal (1), and hydrops fetalis (1) with no other structural abnormality. There were no cases with isolated facial, chest, or spinal anomalies, or raised nuchal translucency $(\mathrm{NT})>4$ $\mathrm{mm}$ (i.e., the minimum measurement required a priori to be eligible for enrollment in the study).

\section{Variant assessment}

After bioinformatic filtering, 22 rare variants relating to 17 potential diagnoses in 15 probands were reviewed by the CRP. In the remaining 12 individuals no candidate pathogenic variants were identified. The mean number of variants identified per case was 1.47 (range 1-3) for the 15 cases with variants to review, and 0.815 across the entire cohort of 27 (Fig. 1). Variants were assessed in 16 different genes known to be associated with various developmental disorders. Of the 15 cases, 13 had variants to review in a single gene (heterozygous [6], compound heterozygous [4], or homozygous [3]) and 2 fetuses harbored homozygous or compound heterozygous variants in two different genes.

\section{Pathogenic variants}

Of the 15 fetuses reviewed by the CRP, 10 were found to be harboring pathogenic/likely pathogenic variants determined to have fully/partially contributed to the phenotype observed on prenatal USS and autopsy, giving a diagnostic rate of $37 \%$ (Table 1). Four of the ten pathogenic variants had arisen de novo (two cases with protein truncating variants in KMT2D, and one case each with missense variants in PIK3R2 and RIT1). The remaining pathogenic variants were inherited, including a maternally inherited FOXP3 missense variant in a 

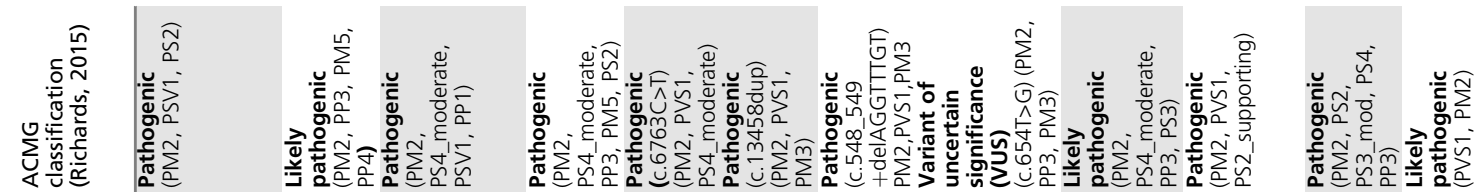

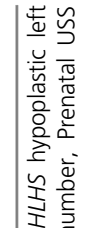

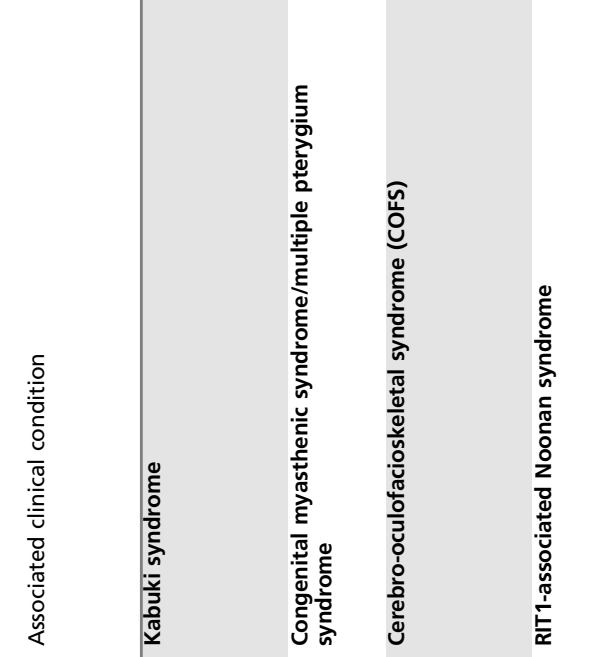

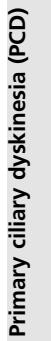
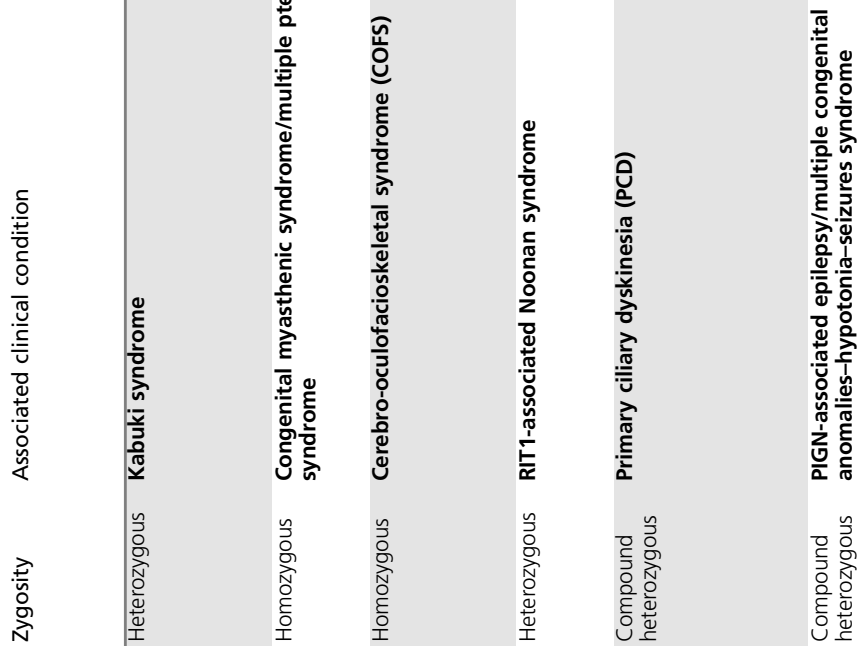

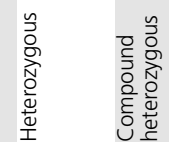

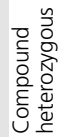

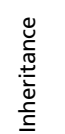

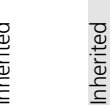

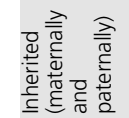

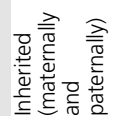

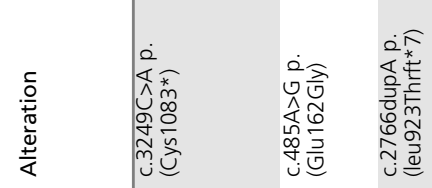

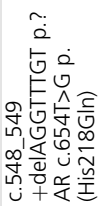

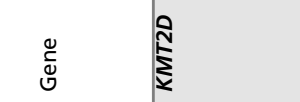

茗

$\frac{2}{2}$

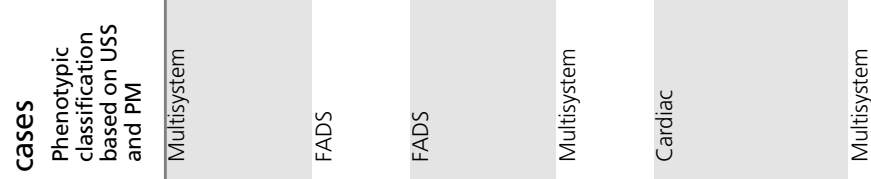

\section{莙}

ํㅡㄹ

일
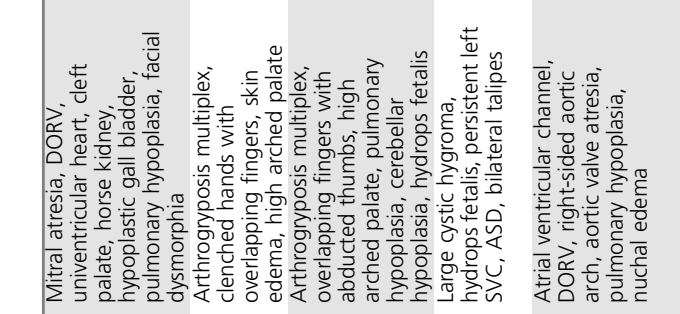

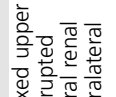

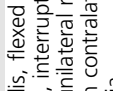

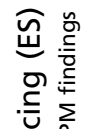

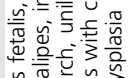

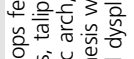

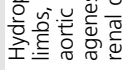

造

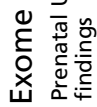

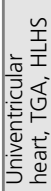

हैํำ

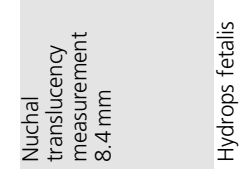

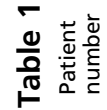

容
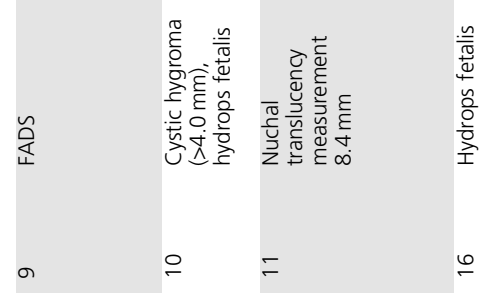
Table 2 Exome sequencing (ES) nondiagnostic cases

\section{Proband Prenatal USS findings}

ID

NT measurement $4.0 \mathrm{~mm}$, arthrogryposis

2

Asymmetrical ventriculomegaly (displacement of the midline), irregularity of the ventricular lining (possibly neuronal heterotopias), possibly absent corpus callosum, abnormal posterior fossa, single outlet to the heart, VSD

\begin{tabular}{ll}
\hline 4 & Short long bones and talipes \\
\hline 5 & Hydrops fetalis \\
\hline $6 \quad \begin{array}{l}\text { Small left ventricle, bilateral superior vena cavas, } \\
\text { levocardia with significant ventricular imbalance, small } \\
\text { slit-like left ventricle with some mitral inflow, } \\
\text { perimembranous VSD, normal large vessels, HLHS } \\
\text { Ebstein-type abnormality, DORV, abnormal left kidney }\end{array}$ \\
$\begin{array}{l}\text { Polyhydramnios, micrognathia, right-side mild dilatation } \\
\text { of the renal pelvis }\end{array}$ \\
\hline $7 \quad$
\end{tabular}

12 Exomphalos, borderline ventriculomegaly

13 Bilateral multicystic, dysplastic kidneys

\begin{tabular}{ll}
\hline 14 & Growth restriction $<10$ th centile \\
\hline 15 Unilateral ventriculomegaly \\
\hline $17 \quad$ Normal \\
\hline $18 \quad \begin{array}{l}\text { Long narrow chest, heart in unusual orientation, single } \\
\text { multicystic dysplastic kidney, unilateral talipes, umbilical } \\
\text { vein varix, minimal liquor }\end{array}$
\end{tabular}

19 Oligohydramnios, unilateral talipes, ?absent bladder and kidneys, IUGR

$20 \quad$ Normal
PM findings

Phenotype

classification

based upon USS

and PM

Skin webs, flexed contractures, severe bilateral talipes,

FADS

webbed neck, micrognathia, reduced muscle bulk, subcutaneous edema

Large cranial vault, TOF, persistent left SVC, right-sided Multisystem aortic arch, absent right umbilical artery, abnormally shaped thymus, severe dysplasia of the cerebellum with obliteration of the 4 th ventricle, malformed brainstem and midbrain, atresia of aqueduct of Sylvius and 4th ventricle, enlarged right cerebral hemisphere, probable arachnoid cyst between hemispheres, abnormal deep white matter bundles in cerebral hemispheres Small size, facial dysmorphia, prominent occiput, bilateral Skeletal talipes, rhizomelic shortening of the limbs, wide-set nipples, osteopenic bones, horizontal ribs Facial dysmorphia, nuchal edema, dilated left atrium and Cardiac left ventricle, cardiomegaly, valvular aortic atresia Overlapping fingers, bilateral talipes, dysmorphic ears, Multisystem skin webbed neck, cardiomegaly, pulmonary stenosis with dysplastic valves, VSD, right ventricular pouch, persistent left SVC, small testis/thymus, ectopic left kidney

Facial dysmorphia, hypoplastic lungs, CPAM-0, small gall Multisystem bladder, small right kidney with dilated renal pelvis, simple renal cysts bilaterally, gracile bones (especially the clavicles, fracture of the right clavicle), excess of extramedullary hematopoiesis in the liver

Omphalocele, bilateral talipes Abdominal

Facial dysmorphia, pterygium of the neck, contractures of Genitourinary the upper limbs, bilateral talipes, transverse palmar creases, bilateral cystic-dysplastic kidneys, lung hypoplasia

Retrognathia, ASD (secundum type), small thymus, lung Cardiac petechiae, distended bladder with retained urine (no evidence of obstruction)

Macrocephaly, partly compressed skull, overlapping

Brain cranial bones, loose scalp, anteverted nares, small heart Micrognathia, omphalocele, bilateral talipes, bilaterally Multisystem three lobed lungs, irregularly shaped left kidney, cervical ribs

Facial dysmorphia, left-side talipes, caudal orientation of Multisystem the heart, aortic valve dysplasia, rectal atresia, multicystic/ aplastic renal dysplasia, lumbar and sacral vertebrae defects

Potter syndrome, bilateral talipes, malpositioned anus,

Multisystem transverse palmar creases (right hand), epicanthus, renal agenesis, unicornuate uterus, lung hypoplasia, irregularities of the sacrum vertebrae Unilateral talipes, complete AV channel, DORV, Multisystem pulmonary stenosis, symmetrical liver, absent gall 
Table 2 continued

\begin{tabular}{|c|c|c|c|}
\hline $\begin{array}{l}\text { Proband } \\
\text { ID }\end{array}$ & Prenatal USS findings & PM findings & $\begin{array}{l}\text { Phenotype } \\
\text { classification } \\
\text { based upon USS } \\
\text { and PM }\end{array}$ \\
\hline & & $\begin{array}{l}\text { bladder, right type isomerism of the lungs, auricles, } \\
\text { absent spleen, right-sided stomach }\end{array}$ & \\
\hline 23 & Polyhydramnios & $\begin{array}{l}\text { Abnormal posture of limbs (flexed legs in the hip and } \\
\text { pointy feet), ulnar deviation of the hands and flexed } \\
\text { thumbs, thin neck and diaphragm, lung hypoplasia, thin } \\
\text { ribs, Arnold-Chiari type II (no cerebellar hypoplasia or } \\
\text { spina bifida) }\end{array}$ & Brain \\
\hline 26 & Congenital bladder neck obstruction & $\begin{array}{l}\text { Subcutaneous edema, scoliosis, talipes and skeletal } \\
\text { abnormalities, distended abdominal wall, anal atresia, } \\
\text { abnormal genitalia, bladder neck obstruction with cystic } \\
\text { bladder, rectal-vesical fistula, small lungs, small kidneys } \\
\text { and adrenals, cystic-dysplastic change in kidneys } \\
\text { associated to LUTO }\end{array}$ & Multisystem \\
\hline 27 & HLHS & HLHS, 11 pairs of ribs, low-set eyes & Cardiac \\
\hline
\end{tabular}

ASD atrial septal defect, AV atrioventricular, CPAM congenital pulmonary airway malformation, DORV double outlet right ventricle, FADS fetal akinesia deformation sequence, HLHS hypoplastic left heart syndrome, IUGR intrauterine growth restriction, LUTO lower urinary tract obstruction, NT nuchal translucency, PM postmortem, SVC superior vena cava, TOF tetralogy of Fallot, USS ultrasound scan, VSD ventricular septal defect.

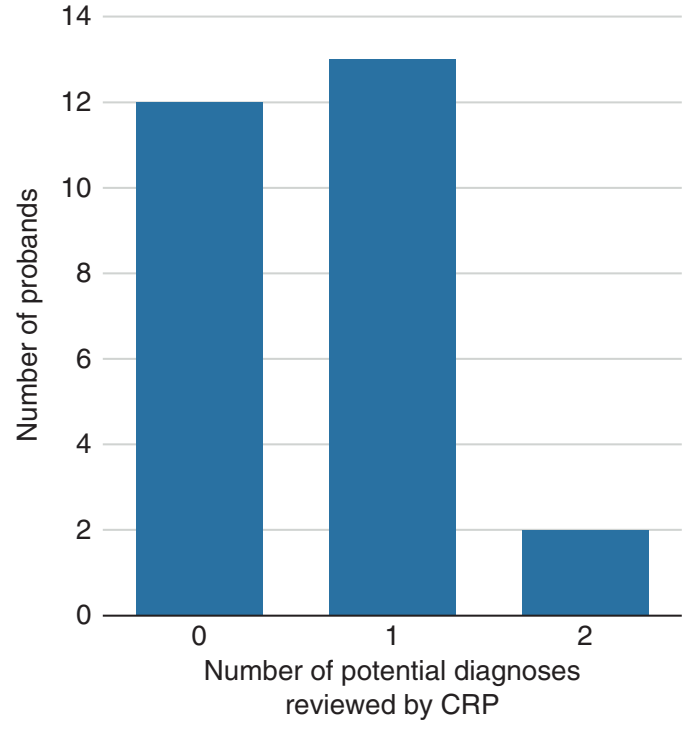

Fig. 1 Number of potential clinical diagnoses per proband reviewed by the clinical review panel (CRP).

male fetus, and three cases with recessively inherited homozygous variants (including a missense variant in RAPSN, and protein truncating variants in ERCC5 and CPT2). The final two cases had biparentally inherited compound heterozygous variants (a paternally inherited missense variant and a maternally inherited protein truncating variant in PIGN, and two different biparentally inherited protein truncating variants in DNAH5). The diagnostic yield across different phenotypic classes is presented in Fig. 2 (based on PM classification). KMT2D gene alterations were determined to be diagnostic in two separate individuals, thus, Kabuki syndrome was the most common diagnosis.

In 6 cases out of the full cohort $(n=27)$, parental consanguinity was recorded. The diagnostic yield was increased in cases with recorded consanguinity $(3 / 6 ; 50 \%)$ as compared with those without consanguinity $(7 / 21 ; 33 \%)$; however this was not statistically significant (Fisher's exact $p$ value $=0.6382$ ). The types of diagnoses did differ however, with all three diagnoses in the consanguineous cases being biparentally inherited homozygous recessive variants, compared with none of the diagnoses in nonconsanguineous cases (Fisher's exact $p$ value $=0.0083$ ).

There were no variants determined to be pathogenic/likely pathogenic but without relevance for the presenting fetal phenotype. In total, ten VUS were identified relating to six individual probands that were determined by the CRP to have no relevance to the fetal phenotype in each case (see Supplementary Table 2). Ethical approval for the study restricted the disclosure of genetic findings to those that were relevant to the FSA and thus these findings were not reported.

\section{DISCUSSION}

The genetic contribution to FSA is incompletely understood, ${ }^{22}$ and evidence relating to the clinical utility of nextgeneration sequencing (NGS) in the investigation of perinatal loss and morbidity in fetuses with structural anomalies is limited. Relatively small cohort studies have been published and indicate that the use of NGS in the perinatal context gives a diagnostic yield of $12-57 \%$ (refs. ${ }^{12,22-24}$ ). However, such publications describe a heterogeneous mix of prenatal ultrasound diagnoses and autopsy findings in fetuses with 


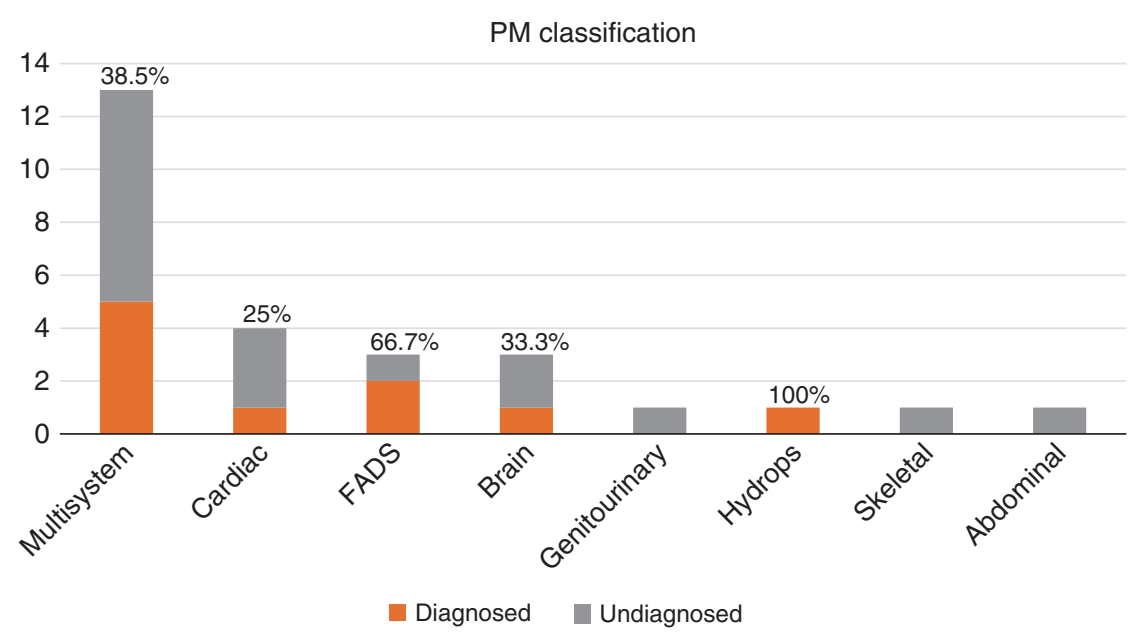

Fig. 2 Diagnostic (pathogenic/likely pathogenic) variant rate according to phenotype classification determined at postmortem examination. FADS fetal akinesia deformation sequence, PM postmortem.

congenital structural anomalies. A recent study investigating FSA described the use of prenatal samples negative for karyotyping and CMA consented for ES, once a parental decision on termination of pregnancy (TOP) was made. Of the 15 fetuses studied, ES identified pathogenic variants in 7 $(47 \%)$ cases providing a likely causative diagnosis. ${ }^{25}$ The largest reported series to date performed ES in 84 deceased fetuses with structural anomalies, 52 of which comprised parental/fetus trios or quads. Where trio data were obtained a diagnostic yield of $24 \%$ was made. In those probands with only fetal DNA tested, there was a lower diagnostic rate of $14 \%\left(\right.$ ref. $\left.{ }^{12}\right)$. In a cohort of perinatal mortality cases $(n=50)$ with limited availability of proband DNA, exome sequencing of parental DNA was performed to identify heterozygous rare variants under a shared model of inheritance. ${ }^{26}$ Utilization of this novel strategy to diagnose recessive monogenic disorders in parents (subsequently confirmed as cosegregating in the affected fetus by targeted testing) demonstrated pathogenic/ likely pathogenic variants in 24 different genes in 26/50 couples $(52 \%)$. Where two or more fetuses were affected a genetic diagnosis was identified in 18/29 couples (62\%), leading the authors to conclude that this is a powerful approach with high clinical utility for genetic diagnosis of lethal or prenatal-onset recessive conditions. Our reported diagnostic yield of $37 \%$ demonstrates further the value of trio ES in combination with a detailed autopsy.

An underlying genetic etiology for the congenital structural anomalies cannot usually be predicted accurately based on the prenatal USS appearances alone. ${ }^{23}$ This is primarily because the phenotype in the developing fetus is often inadequately defined. For example, a recent retrospective study examined the diagnostic utility and limitations of ES in prenatal cases with structural birth defects. DNA from 20 trios (fetal and parental), with normal karyotype and CMA findings, underwent ES and variant interpretation. ${ }^{27}$ The ES results were later reevaluated utilizing details of prenatal and postnatal phenotyping. Initial analysis using only a clinical description of prenatal ultrasound findings revealed no pathogenic/likely pathogenic variants in the 20 pregnancies evaluated but reanalysis with a combination of prenatal and postnatal phenotyping yielded pathogenic variants in at least $20 \%$ of cases.

In our series there was generally a very good association between prenatal USS descriptions and autopsy findings (as previously noted in a larger study from our group $\left.{ }^{6}\right)$. However in four cases (Fig. 2), subtle findings identified on autopsy led to a reclassification of the fetal phenotype. Though such findings do not necessarily influence the working clinical diagnosis (and as appertains to this cohort did not translate into an increased incidence of pathogenic variants), additional information available from autopsy can aid interpretation of ES detected variants and increase diagnostic yield or help exclude variants that come through the bioinformatics filtering process but are unlikely to account for the relevant FSA phenotype. In case 8 a homozygous missense substitution in RAPSN (c.485A>G p.(Glu162Gly) was identified in a fetus with a prenatal USS finding of fetal akinesia deformation sequence and autopsy confirmed the presence features of arthrogryposis multiplex. RAPSN encodes the receptorassociated protein, rapsyn (RAPSN) involved in AChR localization and assembly and localization of the postsynaptic muscle nicotinic acetylcholine receptor. Recessively inherited pathogenic variants in RAPSN were initially described in individuals with congenital myasthenic syndrome ${ }^{28}$ and subsequently with lethal fetal akinesia. ${ }^{29}$ Previously it has been noted that early severe onset disease (fetal akinesia/ multiple pterygium syndrome) tended to be associated with severe loss-of-function pathogenic variants and later-onset myasthenic syndrome with missense substitutions causing milder alterations of function. ${ }^{29,30}$ Though RAPSN missense substitutions (as case 8) have been associated with severe disease the availability of detailed information from the autopsy (including lack of evidence for central nervous system cause) contributed to a consensus classification of the variant as likely pathogenic. In addition the combination of ES and a more detailed autopsy phenotype can expand knowledge of 
the prenatal phenotype of developmental disorders and/or rarer manifestations of severe cases. For example, case 25 was found to have renal cystic dysplasia on prenatal ultrasonography and also demonstrated polymicrogyria of the central nervous system with calcification. ES analysis revealed a homozygous truncating CPT2 variant consistent with a diagnosis of carnitine palmitoyltransferase (CPT) II deficiency. Although this rare disorder typically presents with myoglobinuria in adults or fasting hypoglycemia in children, rarely severe cases may present prenatally. ${ }^{31}$ In a review of 19 such cases, cerebral calcification was noted in 3 cases and polymicrogyria in 1 case. $^{31}$ In cases in which ES was not diagnostic, such as case 23, autopsy examination identified abnormalities that were not identifiable on prenatal ultrasound and this refined the phenotypic abnormalities and might facilitate future diagnosis. It is acknowledged, however, that this cohort of cases were highly selected with a degree of selection bias.

The genetic findings greatly assist assessment of recurrence risk in future pregnancies. In the four cases with de novo findings it was possible to reassure the parents of a low recurrence risk. The parents with inherited diagnoses (6) were counseled as to the likely risk of recurrence according to the mode of inheritance in each individual case (case studies 1 and 2). Those with recurrence risks then had the option for invasive prenatal diagnosis in a subsequent pregnancy to ascertain if the fetus was affected with the same condition.

For the duration of this study the trio samples were reanalyzed at five planned time points (data freezes) to include recently identified genes, as well as new alterations in known genes that associate with congenital malformation (Supplementary Table 1). This resulted in the detection of pathogenic variants in two fetuses that previously lacked a genetic diagnosis, in case 25 (CPT2 stop gained mutation) and case 11 (DNAH5 compound heterozygous frameshift/stop gained mutations). Both genes were added into the virtual gene panel after the initial review of the fetuses, demonstrating the strength of our iterative analysis approach. The added value of systematic review of ES data in the light of new knowledge has been shown to significantly improve diagnostic yield in the context of the Deciphering Developmental Disorders study. ${ }^{17}$ Through improved variant calling methods, novel variant detection algorithms, updated variant annotation, evidence-based filtering strategies, and newly discovered disease-associated genes, diagnostic yield in a pediatric cohort with clinically diagnosed development delay was increased by $13 \%$ (from $27 \%$ to $40 \%$ ). This raises the importance of reanalysis of perinatal sequencing data in relation to ongoing genetic diagnosis, and demonstrates the need for further research as to the potential added value of genomic reanalysis in undiagnosed fetal and neonatal mortality cases.

Our findings therefore support the routine use of molecular autopsy using trio ES and full autopsy to investigate perinatal loss and elucidate the underlying genetic basis of structural developmental abnormality, particularly in relation to severe "lethal" fetal phenotypes where the ethical issues that cause concern with testing in an ongoing pregnancy are somewhat ameliorated in pregnancies that have already ended.

\section{ELECTRONIC SUPPLEMENTARY MATERIAL}

The online version of this article (https://doi.org/10.1038/s41436018-0298-8) contains supplementary material, which is available to authorized users.

\section{ACKNOWLEDGEMENTS}

This publication is part of the PAGE Study and represents independent research commissioned by the Health Innovation Challenge Fund (HICF-R7-396), a parallel funding partnership between the Department of Health and Wellcome Trust. The views expressed in this publication are those of the author(s) and not necessarily those of the Department of Health or Wellcome Trust. E.M. acknowledges support from National Institute for Health Research (NIHR) Cambridge Biomedical Research Centre and a NIHR Senior Investigator Award. The University of Cambridge has received salary support in respect of E.M. from the NHS in the East of England through the Clinical Academic Reserve. The authors would like to thank the families who kindly participated in this research, and acknowledge the PAGE Study Collaborative Group for their support with this publication. E.Q.-J. is funded through the PAGE Study (HICF-R7-396). The PAGE Study HICF-R7-396 grant holders are M.E. Hurles, Wellcome Sanger Institute, Hinxton, Cambridge, UK; M.D. Kilby, West Midlands Fetal Medicine Centre, Birmingham Women's \& Children's NHS Foundation Trust, Birmingham, UK and Institute of Metabolism and Systems Research, College of Medical and Dental Sciences, University of Birmingham, Birmingham, UK; L.S. Chitty, UCL Great Ormond Street Institute of Child Health and North East Thames Regional Genetics Service, Great Ormond Street NHS Foundation Trust, London, UK; E.R. Maher, Department of Medical Genetics, University of Cambridge and NIHR Cambridge Biomedical Research Centre, Cambridge, UK; D. McMullan, West Midlands Regional Genetics Service, Birmingham Women's and Children's Hospital NHS Foundation Trust, Birmingham, UK; L. Jenkins, UCL Great Ormond Street Institute of Child Health and North East Thames Regional Genetics Service, Great Ormond Street NHS Foundation Trust, London, UK; J. Fisher, ARC, London, UK; and M. Parker, Wellcome Centre for Ethics and Humanities and The Ethox Centre, University of Oxford, UK.

\section{DISCLOSURE}

The authors declare no conflicts of interest.

\section{REFERENCES}

1. Persson $M$, Cnattingius $S$, Villamor $E$, et al. Risk of major congenital malformations in relation to maternal overweight and obesity severity: cohort study of 1.2 million singletons. BMJ 2017;357:j2563. On the open access journal it is page $1-8$.

2. Wapner RJ, Martin CL, Levy B, et al. Chromosomal microarray versus karyotyping for prenatal diagnosis. N Engl J Med. 2012;367:2175-2184.

3. Robson SC Chitty LS Ambler G et al. Evaluation of array comparative genomic hybridisation in prenatal diagnosis of fetal anomalies: a 
multicentre cohort assessment of patient, health professional and commissioner preferences for array comparative genomic hybridisation. Southampton UK NIHR Journals Library 2017:4:1-103.

4. Hillman SC, McMullan DJ, Hall G, et al. Use of prenatal chromosomal microarray: prospective cohort study and meta-analysis. Ultrasound Obstet Gynaecol. 2013;41:610-620.

5. Van den Veyver IB Eng CM Genome-wide sequencing for prenatal detection of fetal single-gene disorders. Cold Spring Harb Perspect Med 2015;(5): a023077.

6. Johns $N$, Al-Salti $W$, Cox $P$, et al. A comparative study of prenatal ultrasound findings and post-mortem examination in a tertiary referral centre. Prenat Diagn. 2004;24:339-346.

7. Phadke SR, Gupta A. Comparison of prenatal ultrasound findings and autopsy findings in fetuses terminated after prenatal diagnosis of malformations: an experience of a clinical genetics centre. J Clin Ultrasound. 2010;38:244-249.

8. Yang Y, Muzny DM, Reid JG, et al. Clinical whole-exome sequencing for the diagnosis of Mendelian disorders. N Engl J Med. 2013;369:1502-1511.

9. Culkier HN, Dueker ND, Slifer SH, et al. Exome sequencing of extended families with autism reveals genes shared across neurodevelopmental and neuropsychiatric disorders. Mol Autism. 2014;5:1.

10. Best $S$, Wou K, Vora N, et al. Promises, pitfalls and practicalities of prenatal whole exome sequencing. Prenat Diagn. 2018;38:10-19.

11. Lord J, McMullan DJ, Eberhardt RY, et al. Prenatal exome sequencing analysis in fetal anomalies detected by ultrasonography: the PAGE study. Lancet (in press).

12. Yates CL, Monaghan KG, Copenheaver D, et al. Whole-exome sequencing on deceased fetuses with ultrasound anomalies: expanding our knowledge of genetic disease during fetal development. Genet Med. 2017;19:1171-1178.

13. Wellcome Sanger Institute. Prenatal Assessment of Genomes and Exomes (PAGE) Study. http://www.sanger.ac.uk/science/collaboration/prenatalassessment-genomes-and-exomes-page. Accessed 15 March 2018.

14. Van der Auwera GA, Carneiro MO, Hartl C, et al. From FastQ data to high confidence variant calls: the Genome Analysis Toolkit best practices pipeline. Curr Protoc Bioinformatics. 2013;43:11.10.1-33.

15. Ramu A, Noordam MJ, Schwartz RS, et al. DeNovoGear: de novo indel and point mutation discovery and phasing. Nat Methods. 2013;10:985-987.

16. McLaren W, Gil L, Hunt SE, et al. The Ensembl Variant Effect Predictor. Genome Biol. 2016;17:122.

17. Wright CF, McRae JF, Clayton S, et al. Making new genetic diagnoses with old data: iterative reanalysis and reporting from genome-wide data in 1,133 families with developmental disorders. Genet Med. 2018 January 11; https://doi.org/10.1038/gim2017246.

18. Richards S, Aziz N, Bale D, et al. Standards and guidelines for the interpretation of sequence variants: a joint consensus recommendation of the American College of Medical Genetics and Genomics and the Association for Molecular Pathology. ACMG Laboratory Quality Assurance Committee. Genet Med. 2015;17:405-424.

19. Royal College of Pathologists. G161 guidelines on autopsy practice: fetal autopsy (second trimester fetal loss and termination of pregnancy for congenital anomaly). June 2017. https://www.rcpath.org/ autopsyguidelinesseries. Accessed 19 July 2018.
20. Royal College of Pathologists. G160 guidelines on autopsy practice: fetal autopsy (third trimester antepartum and intrapartum stillbirth). June 2017. https://www.rcpath.org/autopsyguidelinesseries. Accessed 19 July 2018.

21. Sebire NJ, Miller S, Jacques TS, et al. Post-mortem apparent resolution of fetal ventriculomegaly: evidence from magnetic resonance imaging. Prenat Diagn. 2013;33:360-364.

22. Shamseldin HE, Kurdi W, Almusafri $F$, et al. Molecular autopsy in maternal-fetal medicine. Genet Med. 20 July 2017; https://doi.org/ 10.1038/gim.2017.111 [Epub ahead of print].

23. Alamillo $\mathrm{CL}$, Powis $Z$, Farwell $\mathrm{K}$, et al. Exome sequencing positively identified relevant alterations in more than half of cases with an indication of prenatal ultrasound anomalies. Prenat Diagn. 2015;35:1073-1078.

24. Armes JE, Williams M, Price $G$, et al. Application of whole genome sequencing technology in the investigation of genetic causes of fetal, perinatal and early infant death. Paediatr Dev Pathol. 2018;21:54-67.

25. Vora NL, Powell B, Brandt A, et al. Prenatal exome sequencing in anomalous fetuses: new opportunities and challenges. Genet Med. 2017;19:1207-1216.

26. Stals KL, Wakeling M, Baptista J, et al. Diagnosis of lethal or prenatal autosomal recessive disorders by parental exome sequencing. Prenat Diagn. 2018;38:33-43.

27. Aarabi M, Sniezek $\mathrm{O}$, Jiang $\mathrm{H}$, et al. Importance of complete phenotyping in prenatal whole exome sequencing. Hum Genet. 2018;137:175-181.

28. Ohno K, Engel AG, Shen X-M, et al. Rapsyn mutations in humans cause endplate acetylcholine-receptor deficiency and myasthenic syndrome. Am J Hum Genet. 2002;70:875-885.

29. Vogt J, Harrison BJ, Spearman $H$, et al. Mutation analysis of CHRNA1, CHRNB1, CHRND, and RAPSN genes in multiple pterygium syndrome/ fetal akinesia patients. Am J Hum Genet. 2008;82:222-227.

30. Michalk A, Stricker S, Becker J, et al. K. Acetylcholine receptor pathway mutations explain various fetal akinesia deformation sequence disorders. Am J Hum Genet. 2008;82:464-476.

31. Boemer F, Deberg M, Schoos R, et al. Diagnostic pitfall in antenatal manifestations of CPT II deficiency. Clin Genet. 2016;89:193-197.

\section{(i)}

Open Access This article is licensed under a Creative Commons Attribution 4.0 International License, which permits use, sharing, adaptation, distribution and reproduction in any medium or format, as long as you give appropriate credit to the original author(s) and the source, provide a link to the Creative Commons license, and indicate if changes were made. The images or other third party material in this article are included in the article's Creative Commons license, unless indicated otherwise in a credit line to the material. If material is not included in the article's Creative Commons license and your intended use is not permitted by statutory regulation or exceeds the permitted use, you will need to obtain permission directly from the copyright holder. To view a copy of this license, visit http://creativecommons.org/licenses/ by/4.0\%.

(C) The Author(s) 2018 